\title{
Numerical Simulation of an Offset Jet in Bounded Pool with Deflection Wall
}

\author{
Xin Li, Yurong Wang, and Jianmin Zhang \\ State Key Laboratory of Hydraulics and Mountain River Engineering, Sichuan University, Chengdu 610065, China \\ Correspondence should be addressed to Jianmin Zhang; zhangjianmin@scu.edu.cn
}

Received 7 July 2017; Accepted 8 October 2017; Published 31 October 2017

Academic Editor: Ling Qian

Copyright (C) 2017 Xin Li et al. This is an open access article distributed under the Creative Commons Attribution License, which permits unrestricted use, distribution, and reproduction in any medium, provided the original work is properly cited.

\begin{abstract}
The $k-\varepsilon$ turbulent model and VOF methods were used to simulate the three-dimensional turbulence jet. Numerical simulations were carried out for three different kinds of jets in a bounded pool with the deflection wall with angles of $0^{\circ}, 3^{\circ}, 6^{\circ}$, and $9^{\circ}$. The numerical simulation agrees well with the experimental data. The studies show that the length of the potential core zone increases with the increase of the deflection angle. The velocity distribution is consistent with the Gaussian distribution and almost not affected by the deflection angle in potential core zone. The decay rates of flow velocity in the transition zone are 1.195, 1.281, 1.439, and 1.532 corresponding to the unilateral deflection angles, $0^{\circ}, 3^{\circ}, 6^{\circ}$, and $9^{\circ}$, respectively. The decay rates of velocity in the transition zone are 1.928 and 2.835 corresponding to the bilateral deflection angles $3^{\circ}$ and $6^{\circ}$. It is also found that the spread of velocity is stronger in the vertical direction as the deflection angles become smaller. The spread rates of velocity with unilateral deflection wall are higher than those with bilateral deflection walls in the horizontal plane in the pool.
\end{abstract}

\section{Introduction}

Jet is an important motion type of fluid and it can be divided into the laminar jet and the turbulent jet according to its turbulent intensity. In hydraulic engineering, the jets almost belong to the turbulent jets [1]. In more detail, As shown in Figure 1, the jet can be divided into three regions: (I) core region or potential flow zone: the max velocity in this zone still maintains the velocity at the jet exit; (II) transition zone: the velocity in this zone decays rapidly; (III) fully development zone. When it moves into the limited pool, the flow is known as confined jets, such as hydraulics jump for energy dissipation. The flow is called wall jet when the main flow is closed to the boundary. The flow is called offset jet when the jet distances away from the boundary. Rajaratnam [2] has discussed these categories in detail. The hydraulic jump with sudden enlargement and drop was commonly used to be an energy dissipator in hydraulic engineering and it is actually a kind of submerged offset jet. According to the pressure in bottom, floor can be divided into three regions: (1) recirculation region, where jet is not affected by the bottom floor, (2) impingement region, where the jet attaches the bottom floor due to the pressure, and (3) wall jet region, where the flow pattern is similar to the wall jet.

Submerged offset jets occur in many engineering applications, so submerged offset jet has received a lot of attention from researchers. However, there have been fewer investigations of jets with small offset ratios $(S / d<5)$ [3-5]. Nasr and Lai [5] determined the mean velocities and turbulence characteristics of a turbulent plane offset jet with a small offset ratio of 2.125 and a Reynolds number of 11,000 using LDA. Yang and Yeh [6] have numerically studied the field of an offset jet with Reynolds number of 15,000 for offset ratios of 3,7 , and 11. In their work, it was revealed that when the flow is fully developed near the exit and when the walls are far away from the region of concern, zero normal gradient boundary condition could be used at the entrainment and exit boundary. Agelin-Chaab and Tachie [7] reported that, in the early region of the flow development, the Reynolds number and the offset ratio have significant effects on the decay of the maximum mean velocity, but the decay and spread rates were found to be nearly independent of the offset ratio at lager downstream distances. Assoudi et al. [8] had investigated the mean velocity and turbulence characteristics of the turbulent 


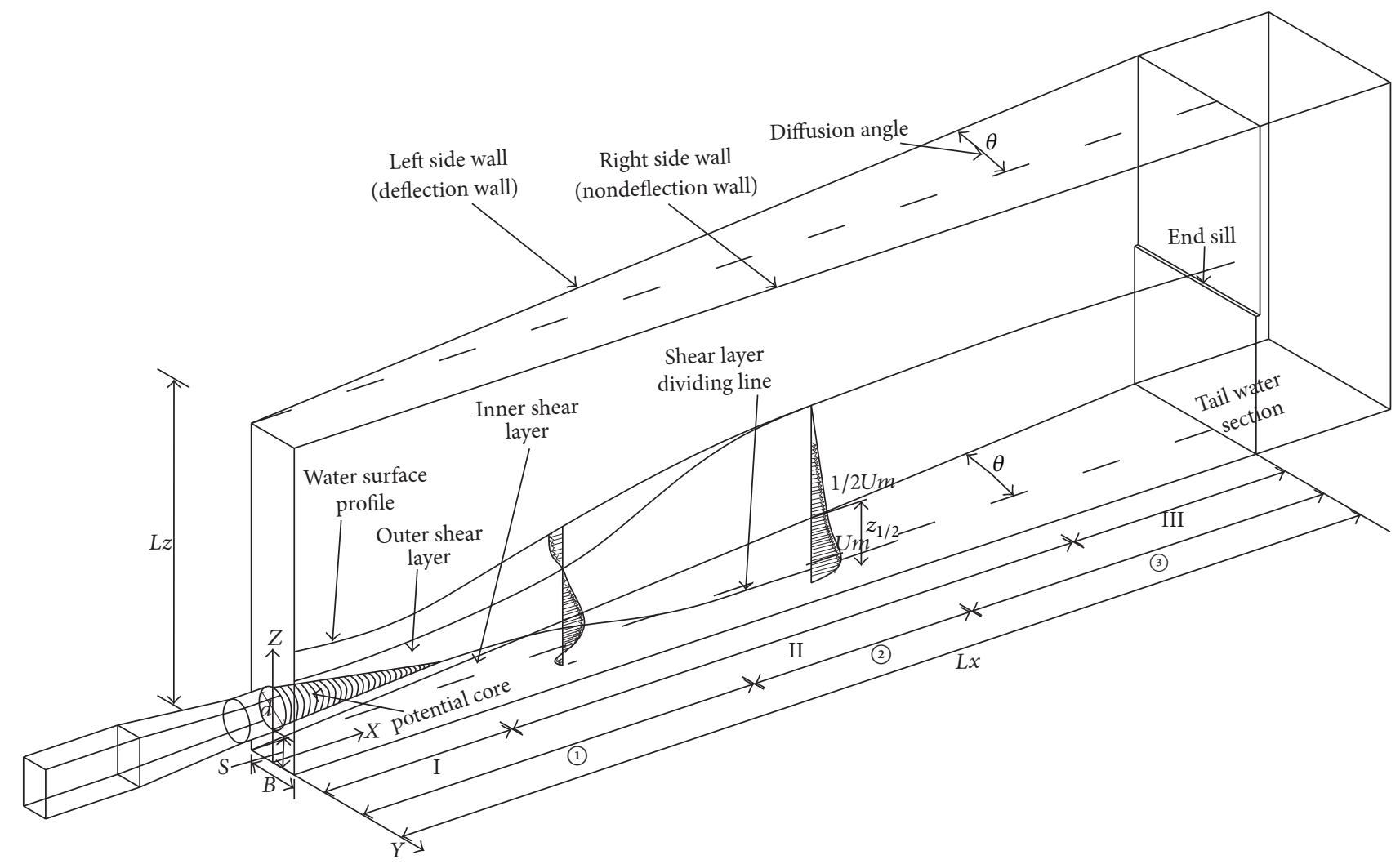

FIGURE 1: The sketch of submerged offset jet inside a unilateral deflection pool. Note. Divided by velocity decay rate in streamwise direction: (I) core region or potential flow zone, (II) transition zone, and (III) full development zone. Divided by pressure in bottom floor: (1) recirculation region, (2) impingement region, and (3) wall jet region.

offset jet using PIV technique at 3 velocity ratios and 2 offset ratios; their results indicated that increasing the offset ratio gives a better distribution of the jet within the flow field, giving rise to a better dynamic mixture. Nyantekyi-Kwakye et al. [9] studied experimentally the jet with different height ratios (0, 2 and 4); their work revealed that the large scale structures within the inner layer increase with wall normal distance from the pool bottom. Further, Rathore and Das [10] reported a numerical investigation for the turbulent offset jet and compared with results proposed by Launder and Sharma [11] and Yang and Shih [12]. They observed a similar profile in the wall jet region due to the resemblance of an offset jet with that of a wall jet flow in the wall jet region. Durand et al. [13] used ADV measuring the velocity of offset jet at the Reynold numbers of $34,000,53,000$, and 86,000; their results indicated the wall normal location of maximum mean velocity and jet spread to be independent of Reynold number. Kishore and Dey [14] provided an experimental investigation of hydraulic characteristics of submerged offset jets by ADV; the streamwise velocity profiles were measured at different sections. The work of Assoudi et al. [15] studied the mean flow behavior of a three-dimensional turbulent offset jet issuing into quiescent ambient; they reported high offset height and it is shown that reattachment point depends strongly on the jet form and the offset height.

In addition, most of the previous studies limit the jet pools to a rectangle. However, sometimes a deflection stilling basin can easily be used to different upstream and downstream conditions; it is more economical than costly transitional structure. The gradually expanding hydraulic jump had been studied by Omid et al. [16]. Besides, Khalif and Moccorquodale [17] carried out a mathematical model of strip integral to investigate radiative hydraulic jump.

With the rapid development of computer power and numerical methods, it is more feasible to use numerical simulation to study the complex turbulent jets. Yue-Tzu Yang (1994), Gu [18], Karim and Ali [19], and Mohammad (2011) show that the $k-\varepsilon$ turbulence model is an effective method to study the jet. In this paper, the standard $k-\varepsilon$ turbulence model and VOF multiphase flow model were used to study the submerged offset jet in no deflection pool, unilateral deflection (deflection angle: $3^{\circ}, 6^{\circ}$, and $9^{\circ}$ ), and bilateral deflection (deflection angle: $3^{\circ}, 6^{\circ}$ ), so the understanding of the flow pattern of the submerged offset jet in a pool with unilateral and bilateral deflection wall can be deepened.

\section{Numerical Model}

2.1. Calculation Model. ANSYS 15.0 ICEM was used to carry out the numerical models for six types of the jet pools, as shown in Table 1 . The computational area contained a circular jet exit, a pool, an end sill, and a tailwater section. The diameter of jet exit is $d=5 \mathrm{~m}$, the offset height is $S=4 \mathrm{~m}$ (offset ratio is $S / d=0.8$ ), the width of the start section of the 
TABLE 1: The jet pool types parameters.

\begin{tabular}{lcc}
\hline Case & Deflection pattern & Deflection angle $\theta$ \\
\hline ND0 & No deflection & $0^{\circ}$ \\
UD3 & Unilateral deflection (left side wall) & $3^{\circ}$ \\
UD6 & Unilateral deflection (left side wall) & $6^{\circ}$ \\
UD9 & Unilateral deflection (left side wall) & $9^{\circ}$ \\
BD3 & Bilateral deflection & $3^{\circ}$ \\
BD6 & Bilateral deflection & $6^{\circ}$ \\
\hline
\end{tabular}

Note. The meanings of the abbreviations in the first column of the table are as follows: $\mathrm{N}$ is non, $\mathrm{U}$ is unilateral, $\mathrm{B}$ is bilateral, $\mathrm{D}$ is deflection, and the number is the deflection angle.

pool is $B=8 \mathrm{~m}$ (expansion ratio $d / B=0.625$ ), the length of the pool is $L x=134.5 \mathrm{~m}$, the height of the pool is $L z=40 \mathrm{~m}$, and the height of the end sill is $\mathrm{He}=17 \mathrm{~m}$. All of cases were meshed using structural grids.

2.2. Governing Equations. The standard $k-\varepsilon$ model was considered to be useful when applied to offset jet flow, Mondal et al. [20], Kumar [21], and Mondal et al. [22]. In addition, Assoudi (2015) believed that the standard $k-\varepsilon$ model is more appropriate for the prediction of the turbulent offset jet with small offset ratio (the offset ratio is 0.8 in this paper). So the standard $k-\varepsilon$ turbulence model presented by Launder and Spalding (1972) was used. The equations of the turbulent kinetic energy, $k$, and its dissipation rate, $\varepsilon$, are as follows:

(1) $k$ equation:

$$
\frac{\partial(\rho k)}{\partial t}+\frac{\partial\left(\rho_{i} k\right)}{\partial x_{i}}=\frac{\partial}{\partial x_{i}}\left[\left(\mu+\frac{\mu_{t}}{\sigma_{k}}\right) \frac{\partial k}{\partial x_{i}}\right]+G_{k}-\rho \varepsilon .
$$

(2) $\varepsilon$ equation:

$$
\begin{aligned}
\frac{\partial(\rho \varepsilon)}{\partial t}+\frac{\partial\left(\rho u_{i} \varepsilon\right)}{\partial x_{i}}= & \frac{\partial}{\partial x_{i}}\left[\left(\mu+\frac{\mu_{t}}{\sigma_{\varepsilon}}\right) \frac{\partial \varepsilon}{\partial x_{i}}\right]+C_{1 \varepsilon} \frac{\varepsilon}{k} G_{k} \\
& -C_{2 \varepsilon} \rho \frac{\varepsilon^{2}}{k} .
\end{aligned}
$$

Here,

$$
\begin{aligned}
u_{t} & =\rho C_{\mu} \frac{k^{2}}{\varepsilon} \\
G_{k} & =\mu_{t}\left(\frac{\partial u_{i}}{\partial x_{j}}+\frac{\partial u_{j}}{\partial x_{i}}\right) \frac{\partial u_{i}}{\partial x_{j}} .
\end{aligned}
$$

When $\rho$ is average density, $u_{i}$ is the average velocity component in the $i$ th direction; $\mu$ is the dynamic viscosity. $C_{\mu}=0.09, \alpha_{k}=1.0, \partial_{\varepsilon}=1.3, C_{1 \varepsilon}=1.44$, and $C_{2 \varepsilon}=1.92$ are empirical constants; $t$ is time, and $P$ is pressure.

The volume of fluid (VOF) was used to track the air-water interface; $\rho$ and $u$ in (1) and (2) are given by the following equation:

$$
\begin{aligned}
& \rho=\alpha_{w} \rho_{w}+\left(1-\alpha_{w}\right) \rho_{a} \\
& \mu=\alpha_{w} \mu_{w}+\left(1-\alpha_{w}\right) \mu_{a}
\end{aligned}
$$

where $\alpha_{w}$ is the volume fraction of water, $\rho_{w}$ and $\rho_{a}$ are the density of water and air, respectively, and $\mu_{w}$ and $\mu_{a}$ are the viscosities of water and air, respectively.

The governing equations were discretized based on the finite volume method using the SIMPLE algorithm for the pressure-velocity coupling. The gradient was calculated by least squares cell-based method. The pressure was discretized by PRESTO! and volume fraction was discretized by GeoReconstruct. The second-order upwind scheme was used for the momentum. The first-order upwind was selected for discretizing the turbulent kinetic energy and the dissipation rate.

\subsection{Boundary Conditions}

(a) Inlet boundary: the inlet is treated as an inlet velocity boundary, where the velocity is set to $30.66 \mathrm{~m} / \mathrm{s}$, so the mean velocity in the jet exit is $37.48 \mathrm{~m} / \mathrm{s}$.

(b) Outlet boundary: the outlet is treated as an outlet pressure boundary.

(c) Wall boundary: it is assumed that there is a no-slip velocity boundary condition; near-wall regions of the velocity are analyzed via the wall function.

(d) Free surface: at the free surface, the inlet pressure boundary is chosen; the pressure value is standard atmospheric pressure.

2.4. Grid Testing. In order to verify the accuracy of the numerical model, the grid convergence index (GCI) (Celik et al., 2008) was selected to test the uncertainty in the numerical results with different grid sizes. The procedure is summarized below.

Step 1. Define a representative grid size $h$.

$$
h=\left[\frac{1}{N} \sum_{i=1}^{N}\left(\Delta V_{i}\right)\right]^{1 / 3}
$$

where $\Delta V_{i}$ is the volume and $N$ is the total number of grids used for computations.

Step 2. Select three significantly different set of grids, $N$, and run simulations to determine the values of key variables, $\phi$. It is desirable that refinement factor $r=h_{\text {coarse }} / h_{\text {fine }}$ be greater 
TABLE 2: Sample calculations of discretization error using the GCI method.

\begin{tabular}{lccc}
\hline & Velocity at $x / d=0.4$ & Velocity at $x / d=6$ & Velocity at $x / d=7.6$ \\
\hline$N_{1}, N_{2}, N_{3}$ & $721854,379432,273439$ & $721854,379432,273439$ & $721854,379432,273439$ \\
$r_{21}$ & 1.9025 & 1.9025 & 1.9025 \\
$r_{32}$ & 1.3876 & 1.3876 & 1.3876 \\
$\phi_{1}$ & 38.3747 & 29.4978 & 18.3938 \\
$\phi_{2}$ & 38.4487 & 28.7691 & 19.9773 \\
$\phi_{3}$ & 38.4645 & 31.9852 & 20.0786 \\
$p$ & 1.0673 & 4.0300 & 2.4936 \\
$\phi_{\text {ext }}^{21}$ & 38.2997 & 29.5568 & 17.9951 \\
$e_{a}^{21}$ & $0.19 \%$ & $2.47 \%$ & $8.61 \%$ \\
$e_{\text {ext }}^{21}$ & $0.20 \%$ & $0.20 \%$ & $2.22 \%$ \\
$\mathrm{GCI}_{\text {fine }}^{21}$ & $0.24 \%$ & $0.25 \%$ & $2.71 \%$ \\
\hline
\end{tabular}

TABLE 3: The parameters of the experiment.

\begin{tabular}{lcccc}
\hline Case & Deflection type & Deflection angle $\theta$ & Mean velocity in jet exit $(\mathrm{m} / \mathrm{s})$ & Reynolds number $(\mathrm{Re}=v d / \nu)$ at jet exit \\
\hline UD6 & Unilateral deflection & $6^{\circ}$ & 37.48 & $1.85 \times 10^{8}$ \\
\hline
\end{tabular}

than 1.3. Therefore, the sizes of the grids were $N_{1}(721,854)$, $N_{2}(379,432)$, and $N_{3}(273,439)$, giving $r$ values of 1.90 and 1.39 .

Step 3. Calculate the apparent order $p$ of the method using the next equation. The equation was solved using fixed point iteration.

$$
\begin{gathered}
p=\frac{1}{\ln \left(r_{1}\right)}|\ln | \varepsilon_{32} / \varepsilon_{21}|+q(p)| \\
q(p)=\ln \left(\frac{r_{21}^{p}-\left[1 \cdot \operatorname{sgn}\left(\varepsilon_{32} / \varepsilon_{21}\right)\right]}{r_{32}^{p}-\left[1 \cdot \operatorname{sgn}\left(\varepsilon_{32} / \varepsilon_{21}\right)\right]}\right),
\end{gathered}
$$

where $\varepsilon_{32}=\phi_{3}-\phi_{2}$ and $\varepsilon_{21}=\phi_{2}-\phi_{1}$.

Step 4. Calculate the extrapolated values from

$$
\phi_{\mathrm{ext}}^{21}=\frac{\left(r_{21}^{p} \phi_{1}-\phi_{2}\right)}{\left(r_{21}^{p}-1\right)}
$$

Step 5. Calculate the approximate relative error $e_{a}^{21}$, extrapolated relative error $e_{\mathrm{ext}}^{21}$, and fine-grid convergence index $\mathrm{CGI}_{\text {fine }}^{21}$ :

$$
\mathrm{GCI}_{\text {fine }}^{21}=\frac{1.25 e_{a}^{21}}{r_{21}^{p}-1}
$$

In this case, the streamwise mean velocity $(U)$ at different positions on the $x$-axis was selected as the variable $\phi$. The sampling point is selected on the jet exit axis, and the $x / d$ range is 0 to 10 due to the high velocity flow in this range which is the focus of this study. Table 2 shows examples of the calculation procedure for the three selected grids. According to Table 1 , the numerical uncertainty in the fine-grid solution for the velocity at $x / d=7.6$ was $2.71 \%$ which corresponded to $\pm 0.50 \mathrm{~m} / \mathrm{s}$.
GCI index values are plotted in the form of error bars, as shown in Figure 2. Based on the grid convergence index, the maximum discretization uncertainty was $7.9 \%$ which corresponded to $\pm 1.73 \mathrm{~m} / \mathrm{s}$. the discretization uncertainty value ranged from $0.01 \%$ to $7.9 \%$, with an average of $1.47 \%$. Based on these results and considering the computational accuracy and time, the number of grids was reasonably set to 379,432 .

2.5. Model Validation. In order to verify the numerical model, the physical model experiments were performed in the State Key Laboratory of Hydraulic and Mountain River Engineering, Sichuan University. The physical model consisted of an upper water tank, a circular tube, a circular jet exit, a pool, an end sill, a tailwater section, a measuring weir, and a reservoir. The physical model scale is $1 / 35$. The size of a jet pool with unilateral $6^{\circ}$ deflection angle was identical to that in the numerical model; other parameters are shown in Table 3. The time average pressure of the bottom floor and the height of water surface had been measured; the layout of the pressure measuring point is shown in Figure 3. It is evident from Figure 4(a) that the water surface height from computation agrees with the corresponding experimental results. Figures 4(b), 4(c), and 4(d) show the pressure values on the bottom of the pool. It can be seen that the numerical simulations were in good agreement with the experimental results; the maximum errors in the pressure were $8.01 \%$. The numerical results thus produced acceptable error values.

\section{Numerical Results and Analysis}

3.1. Flow Pattern. When the jet enters the quiescent body of water, there is a velocity shear between the entering and the ambient water. The velocity shear is unavoidably disturbed during flow, loss of stability, and develops flow oscillations in the shear layer. These oscillations will roll up to form vortexes with increase in size and strength with the axial 


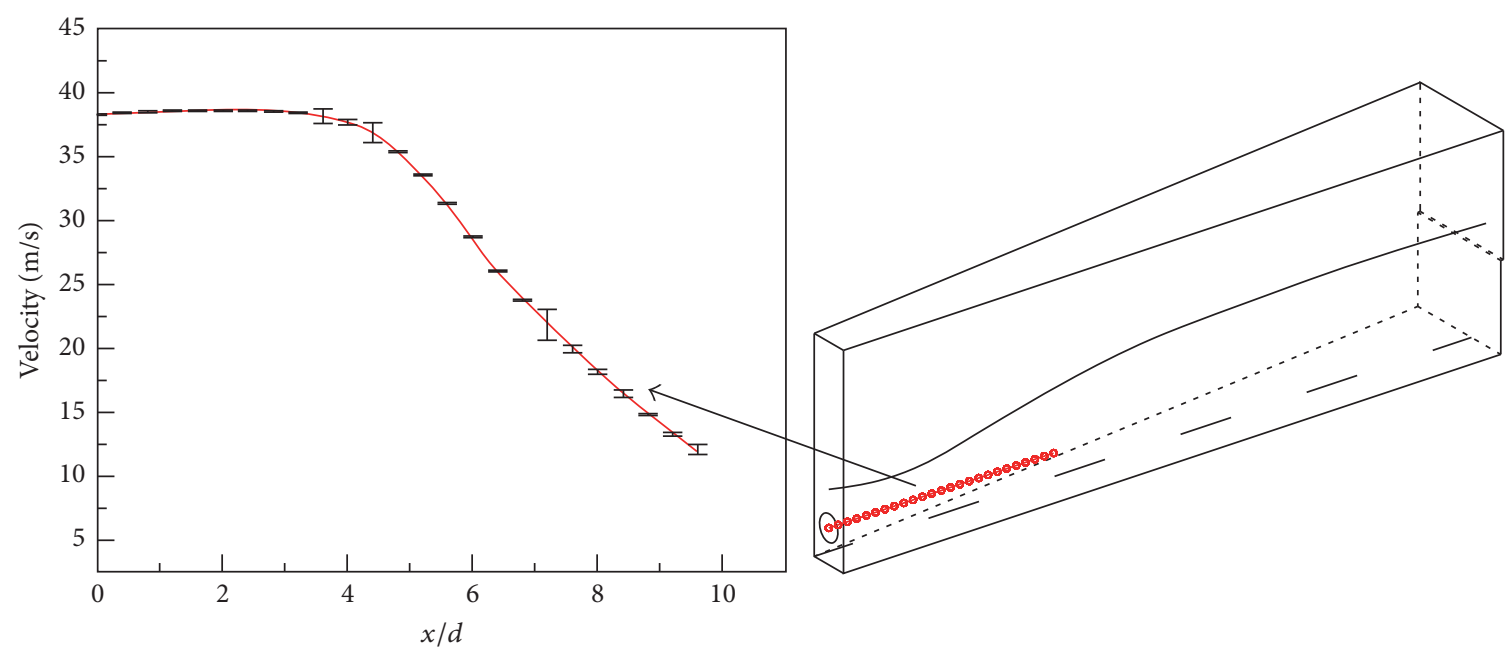

FIgURE 2: Fine-grid solution with discretization error bars computed using the GCI index.

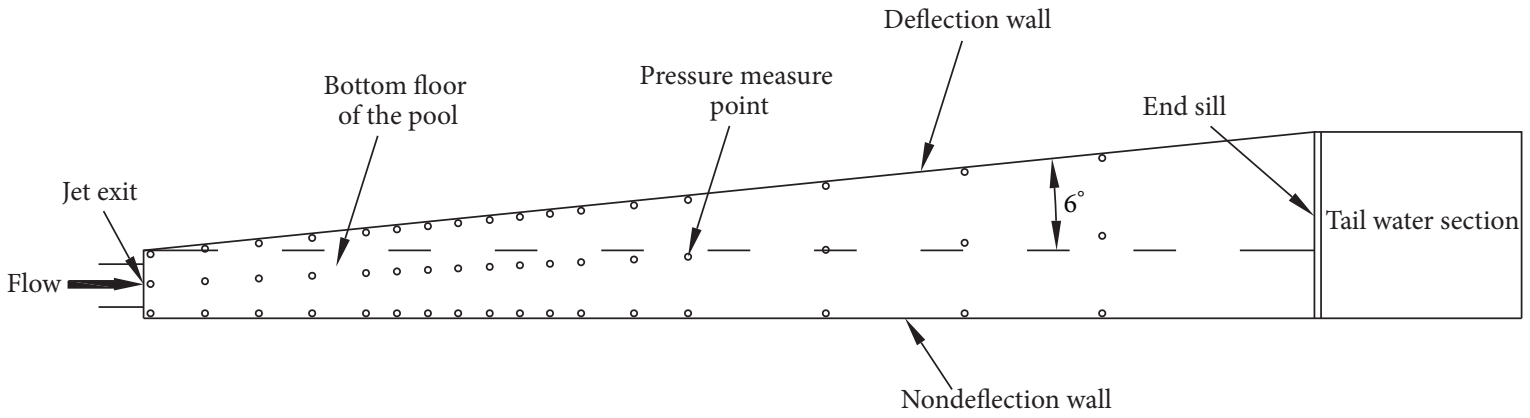

Figure 3: The measured point of time average pressure on the bottom floor in the pool.

distance. The vortexes will influence the entrainment of the ambient water and the mixing of the ambient water and the jet water. (1) For the jet pool with no deflection, the volume of ambient water is relatively small due to the expansion ratio $d / B=0.625$; the spread of the flow section is greatly restricted by the walls on both sides. From Figure 5(a), there is very small vertical axis vortex on both sides of the front part of the jet, then disappearing fast as the flow section spread to the side walls. (2) For the jet pool with unilateral deflection wall, the ambient water on both sides of the jet is asymmetrical; the restriction of the jet flow section on the side of the deflection wall is less compared to the side of the nondeflection wall; there are recirculation zone and the vertical axis vortex due to the jet flow interaction with the ambient water on the side of deflection wall. It is evident from Figures 5(b), 5(c), and 5(d) that the recirculation zone and vertical axis vortex are larger as the deflection angle increase. (3) For the jet pool with bilateral deflection, the process of mixing between flow jet and ambient water less affected by the side walls, the oscillations have enough conditions to produce vortexes, if the deflection angle is large enough. As shown in Figures 5(e) and 5(f) the recirculation zone and vertical axis vortex are symmetrically dispersed on both sides of the jet; the size of the vortexes increases as the deflection angle increases.
3.2. The Velocity Attenuation of Mainstream. Due to the lateral transmission of momentum, the entrained fluid gets momentum and flows forward with the original jet, and the original fluid momentum decreases and the velocities are lost to form a certain velocity gradient. The results of the mixing: the jet flow of section continues to expand, while the velocity is decreasing. In the mainstream direction, the velocity decay can be divided into three zones, including (I) potential core zone-the velocity in this zone still maintains the velocity at the jet exit; (II) transition zone-the velocity in this zone decays rapidly; (III) fully development zone-the velocity in this zone is small.

Figure 6 shows the attenuation of maximum mean velocity $U m$ for all types of jet pool. It can be found that, whether the jet pool is the unilateral deflection or bilateral deflection, the length of the potential core increases as the deflection angle increases. For the same deflection angle, the length of the potential core in the bilateral deflection jet pool is longer than that in unilateral deflection pool. The results show that if the deflection level of the jet pool is higher, flow is less affected by wall restrictions in the potential core zone, indicating that the turbulence intensity of the flow in potential core zone also is smaller, and the energy dissipation effect is worse.

Clark [23] reported that the decaying law of jet mean velocity in the transition zone is $U m \propto(x / d)^{-\eta}$, where $\eta$ 


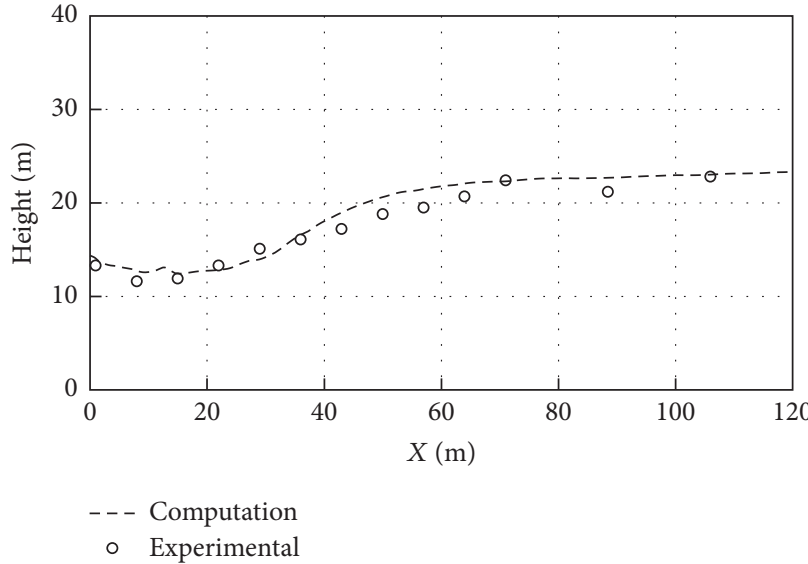

(a) Heights of the water surface in streamwise direction

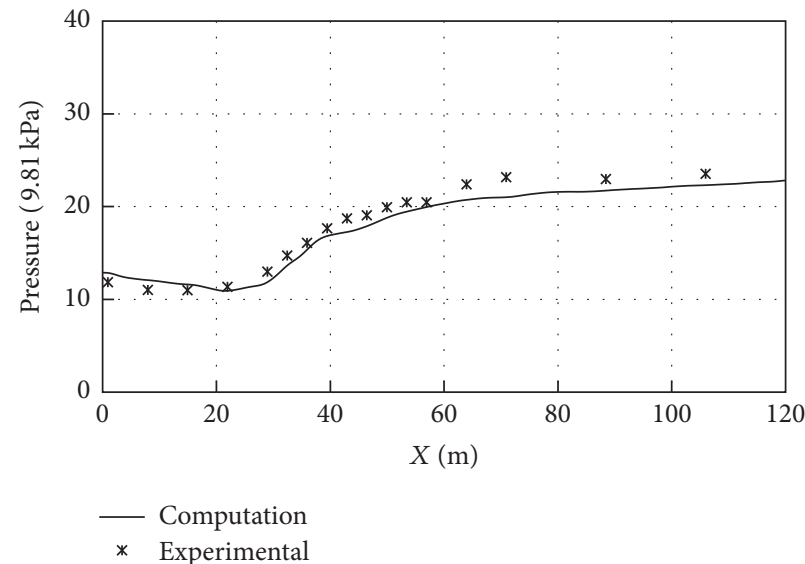

(c) Bottom pressure in the central line

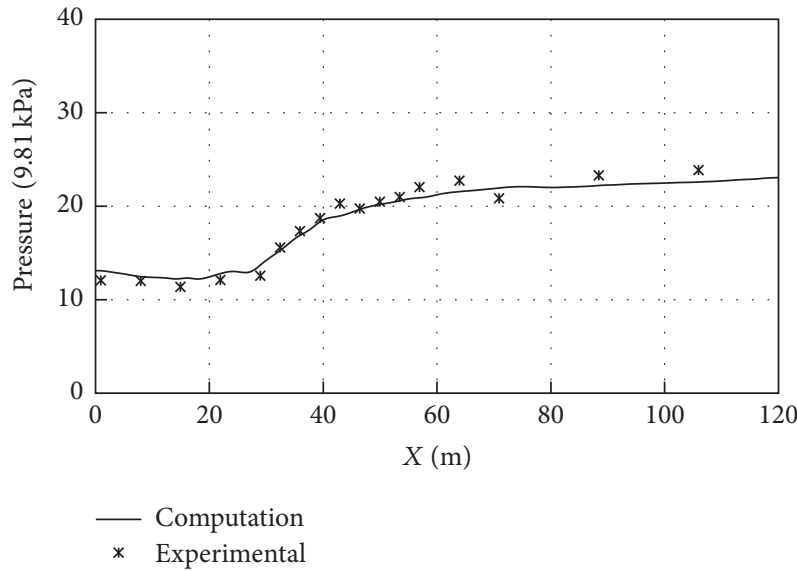

(b) Bottom pressure near the deflection wall

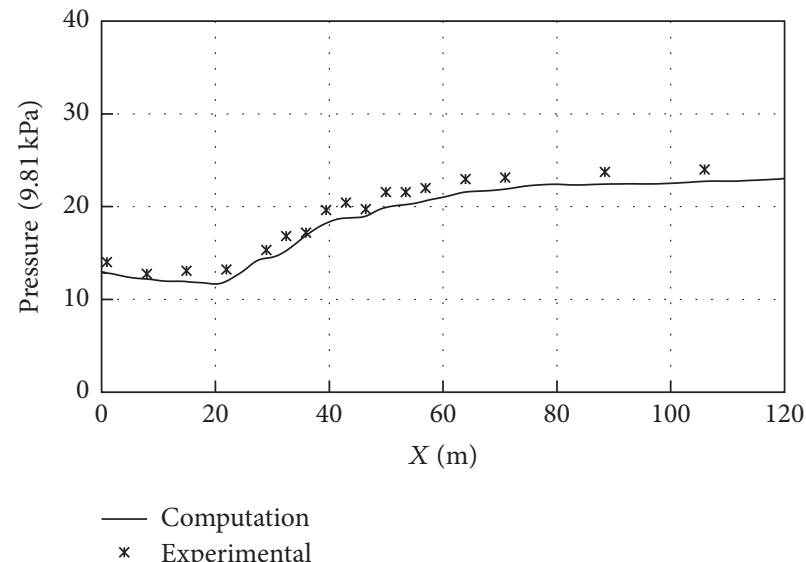

(d) Bottom pressure near the nondeflection wall

FigURE 4: Comparison of numerical and experimental values.

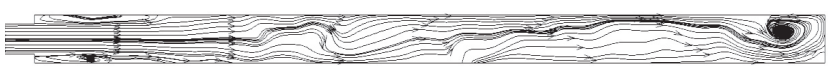

(a) No deflection $0^{\circ}$

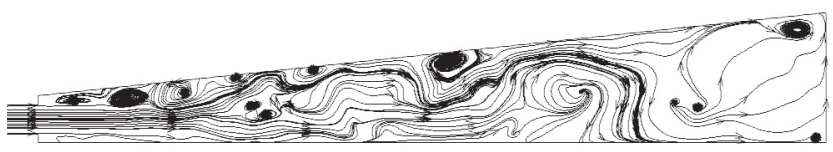

(c) Unilateral deflection $6^{\circ}$

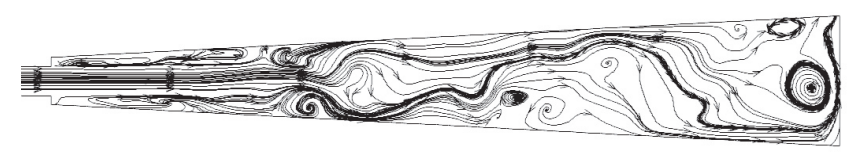

(e) Bilateral deflection $3^{\circ}$

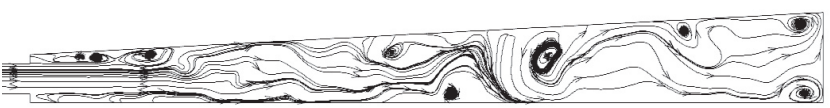

(b) Unilateral deflection $3^{\circ}$

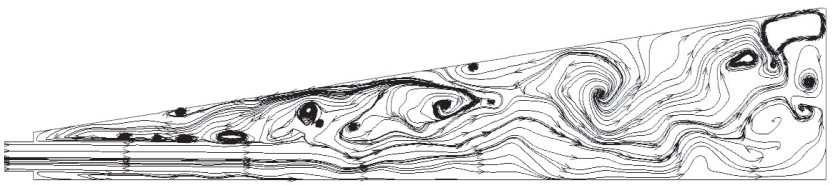

(d) Unilateral deflection $9^{\circ}$

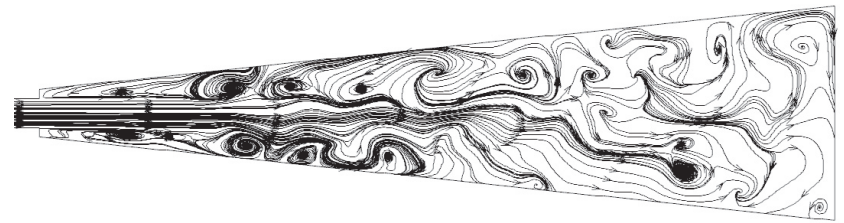

(f) Bilateral deflection $6^{\circ}$

FiguRE 5: Instantaneous streamlines on different deflection patterns and deflection angle of the jet pool. 


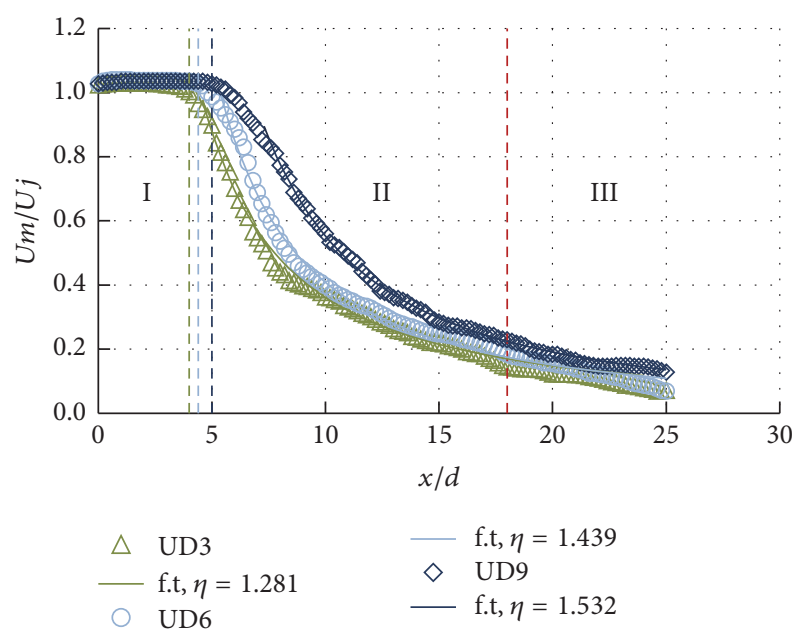

(a) Unilateral deflection

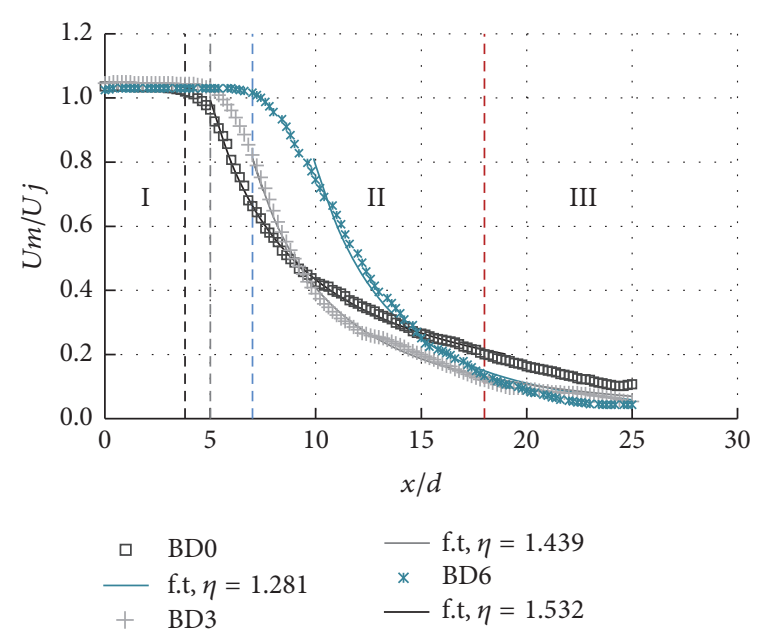

(b) No deflection and bilateral deflection

Figure 6: Distribution of maximum mean velocity for all types of jet pool. Note. The f.t. means fitting curve.

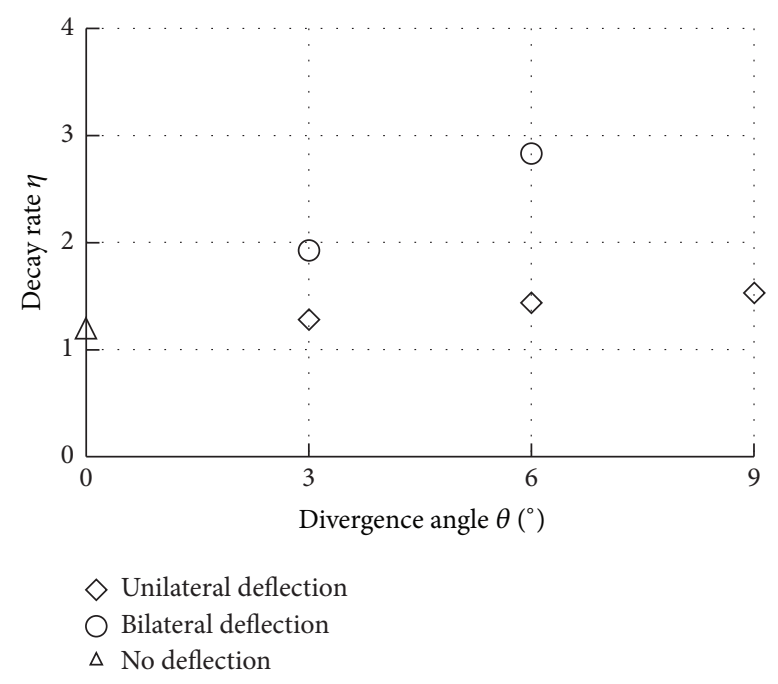

FIGURE 7: The decay rate of mean velocity in transition on different deflection pattern and deflection angle of jet pool.

is called decay rate. Figure 7 shows that, within transition zone, the deflection angle of the pool has significant effect on the decay rate. The decay rate, $\eta$, varies between $1.281 \sim 1.532$ and 1.195 2.835 for unilateral deflection pool and bilateral deflection pool, respectively. The decay rate, $\eta$, is lowest at the no deflection pool and increases with increasing deflection level.

As the flow oscillations will roll up to form vortexes, the kinetic energy from the jet flow is transferred to the turbulent vortexes. As a result, the pool consists of vortexes of different size. This energy is then handed down to smaller and smaller scales through an inviscid process called vortex stretching [24]. At the smallest scales, the vortexes kinetic energy is dissipated by viscous action. For the transition zone, if the deflection level of the jet pool is higher, this indicates that the

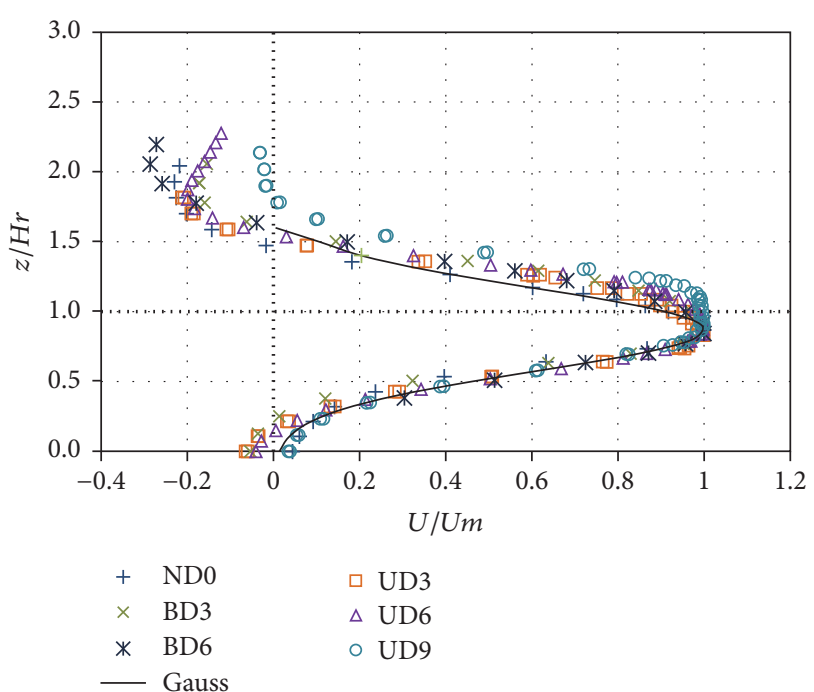

FIGURE 8: Velocity distribution in vertical direction at the end of the potential core. Note. $H r=S+(1 / 2) d$.

deflection level of the pool is higher; the effect of mixing on the velocity decay is more pronounced.

3.3. Velocity Distribution on Vertical Plane. A jet spreads in the vertical directions as it entrains the surrounding fluid. The vertical spread was expanded in both the inner and outer shear layer. It was expected that the pool floor would limit the spread in the inner layer. The velocity distribution in vertical direction at the end of potential core zone with different deflection angles can be seen in Figure 8. It can be found that the velocity distribution in the vertical direction is basically the same at the end of the potential core zone; it conforms with the Gaussian distribution. The results show that the velocity distribution characteristics in the potential core zone have little difference. 


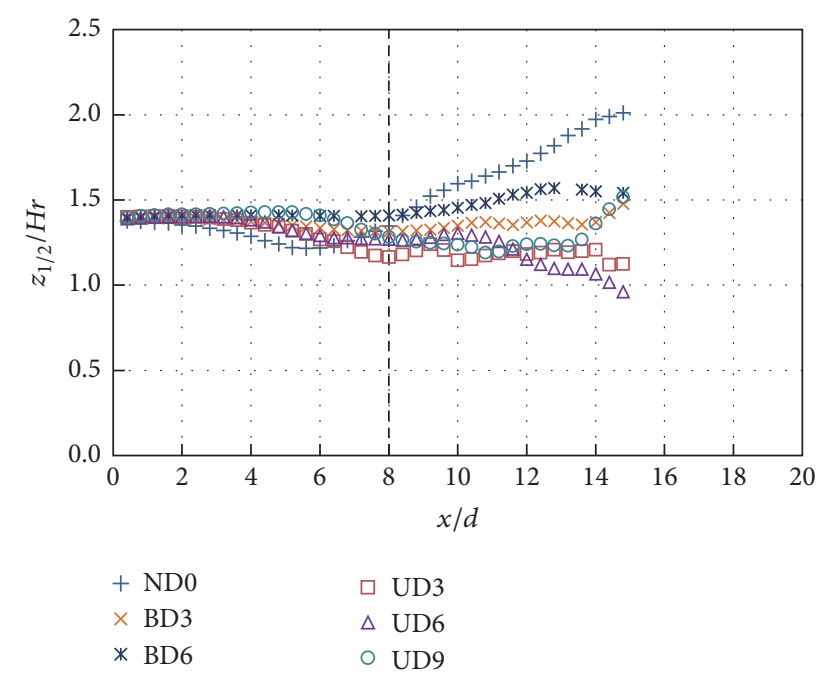

Figure 9: Vertical half-width $\left(Z_{1 / 2}\right)$ distribution along the streamwise direction.

Vertical half-width $\left(Z_{1 / 2}\right)$ distributions along the streamwise direction were shown in Figure 9 and it can be clearly seen that when $x / d \leq 8$, the deflection angle of the jet pool (no deflection, unilateral deflection, and bilateral deflection) has little effect on $Z_{1 / 2}$. Besides, in the potential core zone, the diffusion law of the velocity in the vertical direction is not affected by the deflection angle of the pool. In addition, Figure 9 also shows that when $x / d>8$, the jet flow $Z_{1 / 2}$ (vertical half width) is higher when the pool is with no deflection or bilateral deflection.

Figure 10 shows the distribution of velocity on the bottom floor $(U s)$ of the pools in streamwise direction. It can be seen that $U s$ grows rapidly in $x / d=5 \sim 13$. The max velocity and velocity gradient are shown in Table 4 , for the cases of unilateral deflection; when the unilateral deflection angle is $3^{\circ}, 6^{\circ}$, and $9^{\circ}$, the maximum Us variation is very small, which is $0.29,0.26$, and 0.26 , respectively, and these are similar to the value of pool with no deflection wall. For the cases of bilateral deflection, it is shown that as the deflection angle increases the maximum $U s$ decreases. In terms of gradients of velocity on bottom floor $\left(U^{*}\right)$, for the jet pools with unilateral deflection wall, the maximum $U^{*}$ decreases from 0.12 to 0.04 as the unilateral angle increased from $3^{\circ}$ to $9^{\circ} . U^{*}$ are 0.07 and 0.03 , when the jet pools are with the bilateral deflection angle of $3^{\circ}$ and $9^{\circ}$, respectively. $U^{*}$ obtained in this paper is within the range of $0.03 \leq U^{*} \leq 0.16$.

The variation rules of maximum $U s$ and the maximum $U^{*}$ reflect the jet flow spread in the vertical direction. The above results indicate that the deflection angle has significant effect on the decay rate. This indicates the deflection angle and the jet spread in the inner layer have strongly contact; the larger the deflection level, the higher the lateral spread rate of jet flow; the corresponding vertical spread rate is weakened.

3.4. Velocity Distribution on Horizontal Plane. The velocity in lateral distribution at the end of potential core zone with different deflection angle was shown on Figure 11(a). The

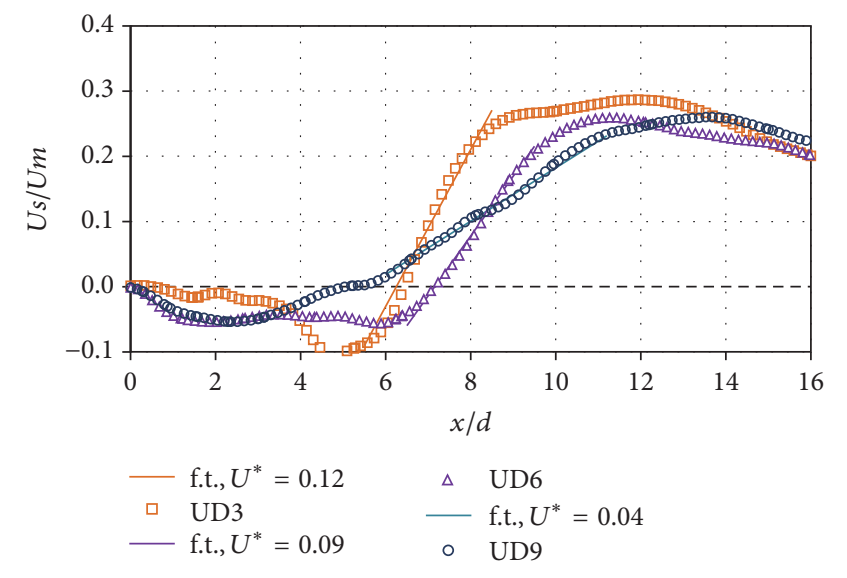

(a) Unilateral deflection

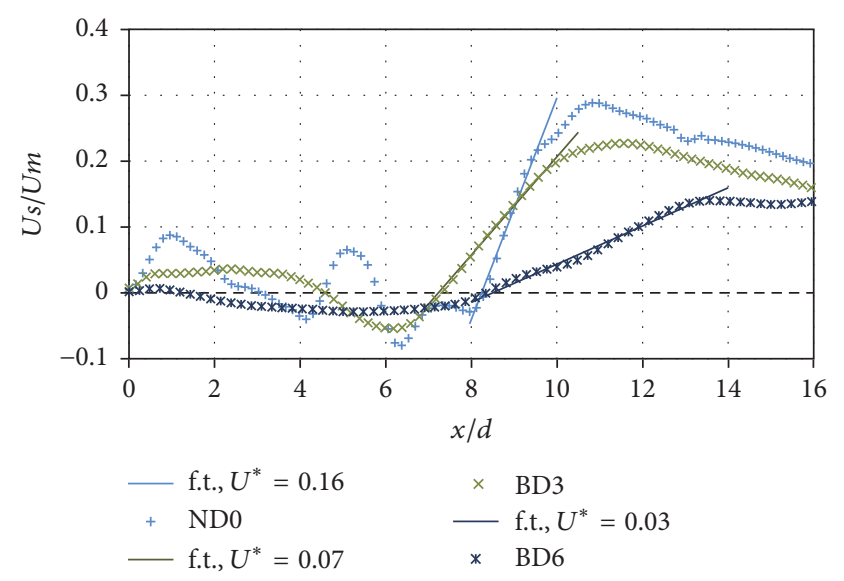

(b) No deflection and bilateral deflection

Figure 10: The distribution of velocity on the bottom floor $(U s)$ of the pools in streamwise direction. Note. The f.t. means fitting curve; and $U^{*}=d(U s / U m) / d(x / d)$.

velocity distribution in the horizontal direction for different cases is substantially the same at the end of the potential core zone and conforms to the Gaussian distribution. This indicates that the deflection wall has no significant effect on the jet spread in horizontal direction when the jet is still in the potential core zone.

Figure 11(b) shows the distribution of velocity on both side walls $(U w)$ of the pools in streamwise direction (unilateral deflection); Table 5 shows the maximum $U w$ and the corresponding location for all cases. The studies show that the jet pool is in the form of unilateral deflection; the maximum velocity $U w$ at the right side wall during the change of the deflection angle from $3^{\circ}$ to $9^{\circ}$ is always relatively higher than nondeflection wall. It is shown that the one side wall deflection of the pool has a little effect on the lateral spread rate of anther side. Figure 11(c) shows the distribution of velocity on both side walls $(U w)$ of the pools in streamwise direction (bilateral deflection); it is obvious that the jet flows attach the wall which need more distance from jet exit as the deflection angle increases; at the same time, the maximum $U w$ decreases as the deflection angle increases. Figure 11(d) compared the velocity in the deflection wall 
TABLE 4: The max velocity and the max velocity gradient on the bottom floor of pools.

\begin{tabular}{lcc}
\hline Case & Max velocity on bottom floor $(U s / U m)$ & Max velocity gradient $U^{*}$ \\
\hline ND0 & 0.30 & 0.16 \\
UD3 & 0.29 & 0.12 \\
UD6 & 0.26 & 0.09 \\
UD9 & 0.26 & 0.04 \\
BD3 & 0.23 & 0.07 \\
BD6 & 0.14 & 0.03 \\
\hline
\end{tabular}

TABLE 5: The maximum $U w$ and the corresponding location for all test cases.

\begin{tabular}{|c|c|c|c|c|}
\hline \multirow{2}{*}{ Case } & \multicolumn{2}{|c|}{ Right side wall } & \multicolumn{2}{|c|}{ Left side wall } \\
\hline & $\operatorname{Max}(U w / U j)$ & Location $(x / d)$ & $\operatorname{Max}(U w / U j)$ & Location $(x / d)$ \\
\hline ND0 & 0.39 & 6.4 & 0.38 & 6.8 \\
\hline ND3 & 0.34 & 8.0 & 0.27 & 7.6 \\
\hline UD6 & 0.32 & 8.8 & 0.13 & 10.0 \\
\hline UD9 & 0.38 & 10.4 & 0.06 & 19.2 \\
\hline BD3 & 0.18 & 10.0 & 0.20 & 11.6 \\
\hline BD6 & 0.03 & - & 0.06 & - \\
\hline
\end{tabular}

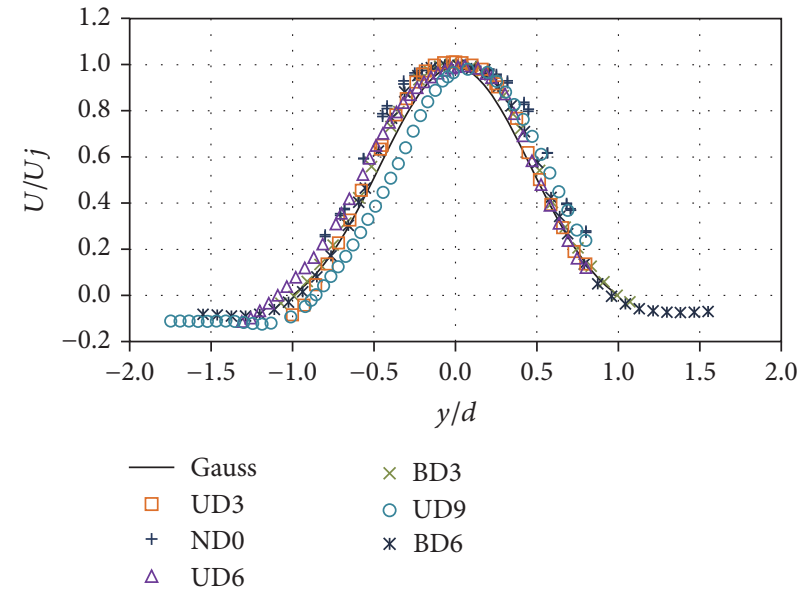

(a)

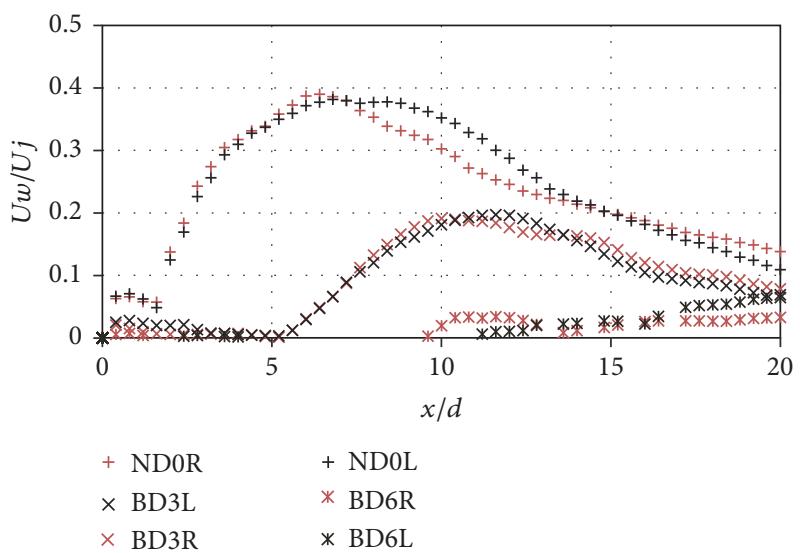

(c)
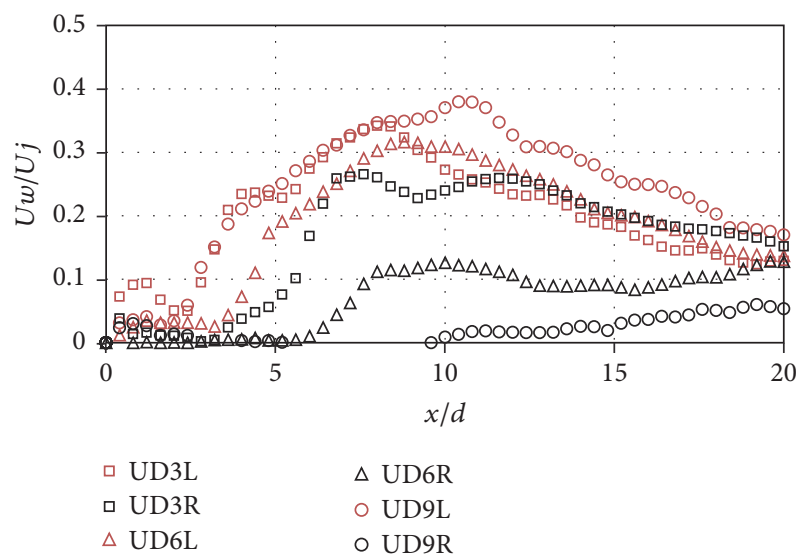

(b)

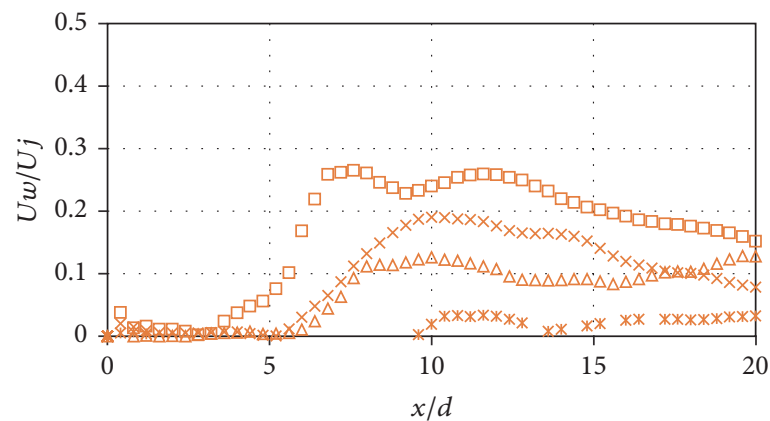

$\begin{array}{ll}\square \mathrm{UD} 3 \mathrm{R} & \triangle \mathrm{UD} 6 \mathrm{R} \\ \times \mathrm{BD} 3 \mathrm{R} & * \mathrm{BD} 6 \mathrm{R}\end{array}$

(d)

FIGURE 11: (a) Velocity $U$ distribution in horizontal direction at the end of the potential core. (b) Distribution of velocity on the both side wall $(U s)$ of the pools in streamwise direction (unilateral deflection). (c) Distribution of velocity on both side walls (Us) of the pools in streamwise direction (bilateral deflection). (d) Comparing the velocity in the deflection wall between unilateral deflection pool and bilateral deflection pools. Note. The R means the right side wall, and the L means the left side wall. 
between unilateral deflection pools and bilateral deflection pools; when the deflection angles of unilateral deflection and bilateral deflection are the same, $U w$ in unilateral deflection pools is higher than that of bilateral deflection. It is clear that when the jet is in the bilateral deflection pool the surrounding water on both sides of the jet is about twice as much as water in the unilateral deflection pool. As a result, the jet section is larger in bilateral deflection pool and velocity decays faster. This indicates that the spread rates of velocity with unilateral deflection wall are higher than those with bilateral deflection walls in the horizontal plane in the pool.

\section{Conclusions}

The jets in the pool with nondeflection wall, unilateral deflection wall $\left(3^{\circ}, 6^{\circ}\right.$, and $\left.9^{\circ}\right)$, and bilateral deflection wall $\left(3^{\circ}, 6^{\circ}\right)$ had been studied in terms of decaying and spreading of the mean velocity. The main conclusions have been drawn as follows:

(1) For the unilateral deflection pool, there are vertical axis vortexes near the deflection wall. For the jet pool with bilateral deflection, the vertical axis vortexes symmetrically dispersed on both sides of the jet and the vertical axis vortexes process more symmetrical feature with the increase of angle. The sizes of these vortexes increase as the deflection angle increases.

(2) As the deflection angle of the jet pool increases, the potential core zone will become longer. For the same deflection angle, the potential core zone in the jet pools with bilateral deflection wall is longer than that in the pool with unilateral deflection angle. In the transition zone, the velocity decay rate $\eta$ in the bilateral deflection pool is larger than that in the unilateral deflection pool.

(3) In the potential core zone, the velocity distributions in the vertical and horizontal directions are consistent with the Gaussian distribution and not affected by the deflection angle. When the jet pool is in the form of unilateral deflection, the flow velocity characteristics on the side of the nondeflection side wall cannot be improved. Compared to bilateral deflection, the jet spread rate of the unilateral deflection is higher in the horizontal direction.

For further understanding the characteristics of offset jet in bounded pool with deflection wall, the Reynolds number, offset ratios, expansion ratio, submergence ratios, and the shape of the jet exit should act as the variables to analyze. Furthermore, the fluctuating pressure of the floor and side walls should be studied through experiments to understand the vortexes characteristics in the pool.

\section{Notation}

B: The width of front part of the pool in $Y$ direction

$d$ : Circular jet diameter $(\mathrm{m})$

$h$ : Representative grid size

$h t$ : Tailwater depth $(\mathrm{m})$

$p$ : The apparent order

$r$ : Refinement factor

$e$ : Error
He: The height of the end sill

$L x$ : The length of the pool in $X$ direction

$L z: \quad$ The height of the pool in $Z$ direction

$V: \quad$ The volume of computation domain

$U: \quad$ Streamwise mean velocity $(\mathrm{m} / \mathrm{s})$

$U j: \quad$ Average of mean jet exit velocity $(\mathrm{m} / \mathrm{s})$

Um: Local streamwise maximum mean velocity $(\mathrm{m} / \mathrm{s})$

Us: $\quad$ The streamwise velocity in the bottom floor of pool $(\mathrm{m} / \mathrm{s})$

$U w: \quad$ The streamwise velocity in the wall of pool $(\mathrm{m} / \mathrm{s})$

$U^{*}: \quad$ The gradient of $U s$

$N$ : The total number of the grids

$X: \quad$ Streamwise direction (m)

$Y: \quad$ Horizontal direction $(\mathrm{m})$

$Z: \quad$ Vertical direction $(\mathrm{m})$

$Z_{1 / 2}$ : Vertical half width $(\mathrm{m})$

$\mathrm{Zm}$ : Lateral location of $U m(\mathrm{~m})$

$S: \quad$ Offset height for jet $(\mathrm{m})$

Re: Reynolds number

$\phi: \quad$ Variables

$\nu$ : Kinematic viscosity $\left(\mathrm{cm}^{2} / \mathrm{s}\right)$

$\theta: \quad$ Deflection angle

$\eta$ : $\quad$ Decay rate

$\mathrm{GCI}_{\text {fine }}^{21}$ : Fine-grid convergence index.

\section{Conflicts of Interest}

The authors declare that they have no conflicts of interest.

\section{Authors' Contributions}

Xin Li and Yurong Wang contributed equally to this work.

\section{Acknowledgments}

This study was funded by the National Natural Science Foundation of China (Grant no. 51579165).

\section{References}

[1] N. Rajaratnam, “The hydraulic jump as a wall jet," Journal of Hydraulics Division, vol. 91, no. 5, pp. 107-132, 1965.

[2] N. Rajaratnam, “Turbulent jets," Developments in Water Science, vol. 5, no. 76, pp. 77-82, 1976.

[3] N. Gao and D. Ewing, "Experimental investigation of planar offset attaching jets with small offset distances," Experiments in Fluids, vol. 42, no. 6, pp. 941-954, 2007.

[4] T. S. Lund, "Augmented thrust and mass flow associated with two-dimensional jet reattachment," AIAA Journal, vol. 24, no. 12, pp. 1964-1970, 1986.

[5] A. Nasr and J. C. S. Lai, "A turbulent plane offset jet with small offset ratio," Experiments in Fluids, vol. 24, no. 1, pp. 47-57, 1998.

[6] Y.-T. Yang and Y.-Y. Yeh, "Numerical study of turbulent offset jets with entrainment boundary," Journal of Thermophysics and Heat Transfer, vol. 8, no. 1, pp. 182-184, 1994. 
[7] M. Agelin-Chaab and M. F. Tachie, "Characteristics and structure of turbulent 3D offset jets," International Journal of Heat and Fluid Flow, vol. 32, no. 3, pp. 608-620, 2011.

[8] A. Assoudi, S. Habli, N. M. Saïd, H. Bournot, and G. L. Palec, "Experimental and numerical study of an offset jet with different velocity and offset ratios," Engineering Applications of Computational Fluid Mechanics, vol. 9, no. 1, pp. 490-512, 2015.

[9] B. Nyantekyi-Kwakye, M. F. Tachie, S. P. Clark, J. Malenchak, and G. Y. Muluye, "Experimental study of the flow structures of 3D turbulent offset jets," Journal of Hydraulic Research, vol. 53, no. 6, pp. 773-786, 2015.

[10] S. K. Rathore and M. K. Das, "Comparison of two low-Reynolds number turbulence models for fluid flow study of wall bounded jets," International Journal of Heat and Mass Transfer, vol. 61, no. 1, pp. 365-380, 2013.

[11] B. E. Launder and B. I. Sharma, "Application of the energydissipation model of turbulence to the calculation of flow near a spinning disc," International Communications in Heat and Mass Transfer, vol. 1, no. 2, pp. 131-137, 1974.

[12] Z. Yang and T. H. Shih, "New time scale based k-epsilon model for near-wall turbulence," AIAA Journal, vol. 31, no. 7, pp. 11911198, 1993.

[13] Z. M. J. Durand, S. P. Clark, M. F. Tachie, J. Malenchak, and G. Muluye, "Experimental Study of Reynolds Number Effects on Three-Dimensional Offset Jets," in Proceedings of the ASME 2014 4th Joint US-European Fluids Engineering Division Summer Meeting collocated with the ASME 2014 12th International Conference on Nanochannels, Microchannels, and Minichannels, pp. V01C-15, 2014.

[14] G. Kishore and S. Dey, "Hydraulics of submerged offset-jets," in Proceedings of the Hydraulic Structures and Water System Management. 6th IAHR International Symposium on Hydraulic Structures, B. Crookston and B. Tullis, Eds., pp. 407-416, 2016.

[15] A. Assoudi, S. Habli, N. M. Saï, P. Bournot, and G. L. Palec, "Numerical study of a turbulent offset jet flow," Lecture Notes in Mechanical Engineering, vol. 789, pp. 703-711, 2017.

[16] M. H. Omid, M. Esmaeeli Varaki, and R. Narayanan, "Gradually expanding hydraulic jump in a trapezoidal channel," Journal of Hydraulic Research, vol. 45, no. 4, pp. 512-518, 2007.

[17] A. A. M. Khalifa and J. A. McCorquodale, "Simulation of the radial hydraulic jump," Journal of Hydraulic Research, vol. 30, no. 2, pp. 149-163, 1992.

[18] R. Gu, "Modeling two-dimensional turbulent offset jets," Journal of Hydraulic Engineering, vol. 122, no. 11, pp. 617-624, 1996.

[19] O. A. Karim and K. H. M. Ali, "Prediction of flow patterns in local scour holes caused by turbulent water jets," Journal of Hydraulic Research, vol. 38, no. 4, pp. 279-287, 2000.

[20] T. Mondal, A. Guha, and M. K. Das, "Computational study of periodically unsteady interaction between a wall jet and an offset jet for various velocity ratios," Computers \& Fluids, vol. 123, pp. 146-161, 2015.

[21] A. Kumar, "Mean flow characteristics of a turbulent dual jet consisting of a plane wall jet and a parallel offset jet," Computers \& Fluids, vol. 114, pp. 48-65, 2015.

[22] T. Mondal, A. Guha, and M. K. Das, "Effect of bottom wall proximity on the unsteady flow structures of a combined turbulent wall jet and offset jet flow," European Journal of Mechanics - B/Fluids, vol. 57, pp. 101-114, 2016.

[23] S. P. Clark, "Flow characteristics within the recirculation region of three-dimensional turbulent offset jet," Journal of Hydraulic Research, vol. 53, no. 2, pp. 230-242, 2015.
[24] S. B. Pope, Modeling Mixing and Reaction in Turbulence Combustion, May 2000, https://www.researchgate.net/publication/ 235185542_Modeling_Mixing_and_Reaction_in_Turbulence_ Combustion. 


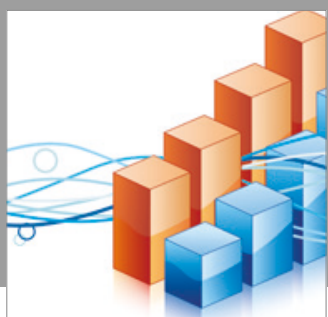

Advances in

Operations Research

vatersals

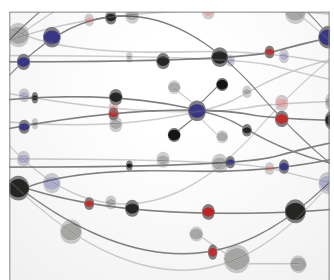

\section{The Scientific} World Journal
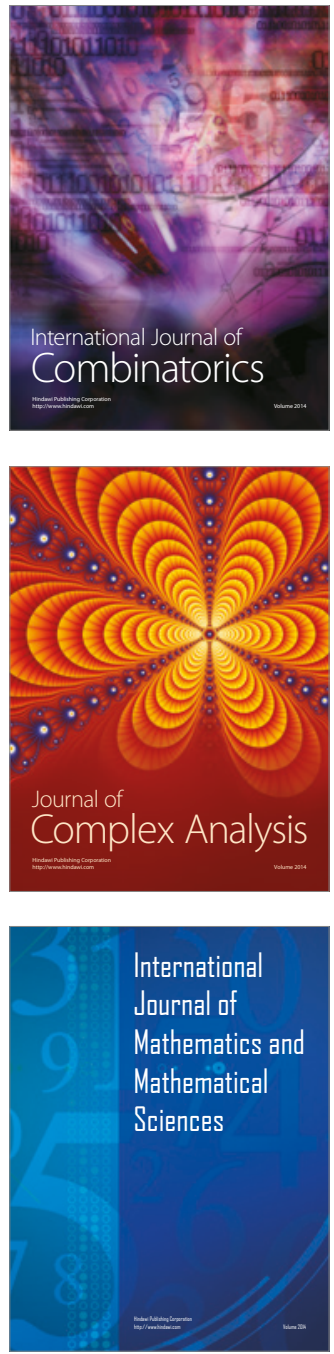
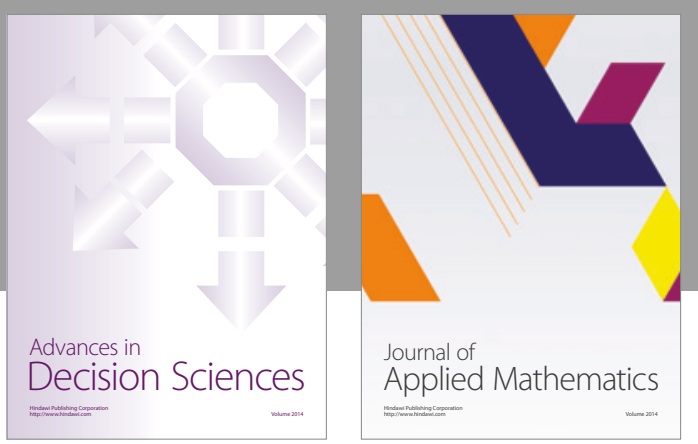

Algebra

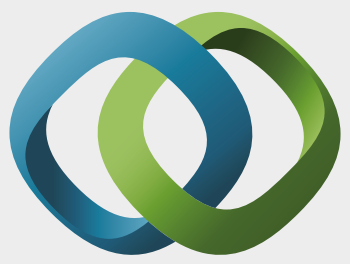

\section{Hindawi}

Submit your manuscripts at

https://www.hindawi.com
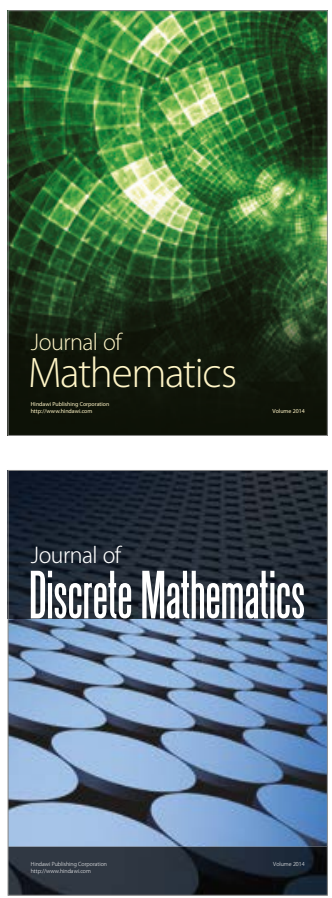

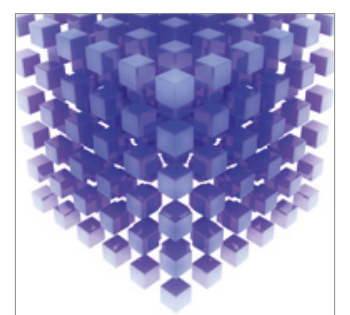

Mathematical Problems in Engineering
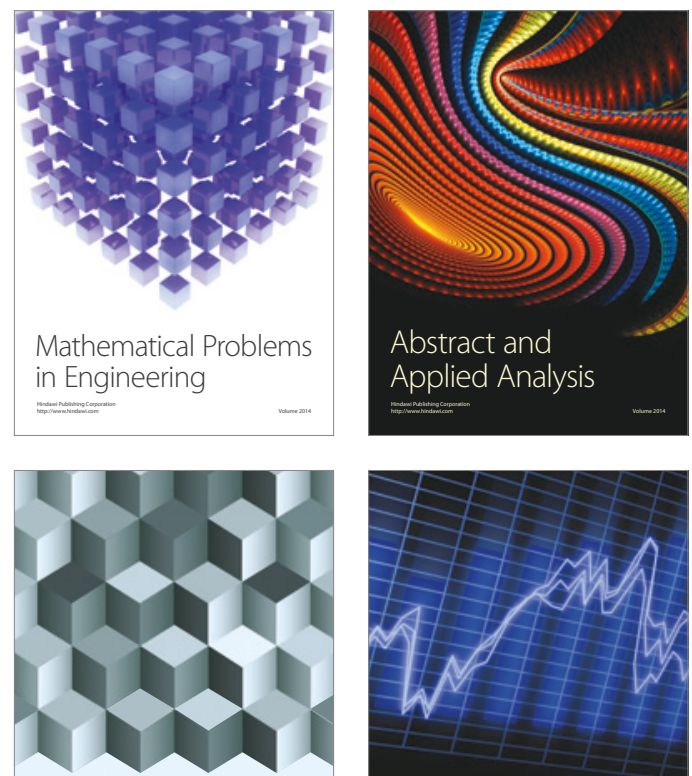

Journal of

Function Spaces

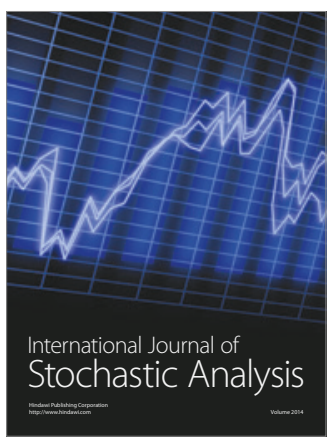

Probability and Statistics
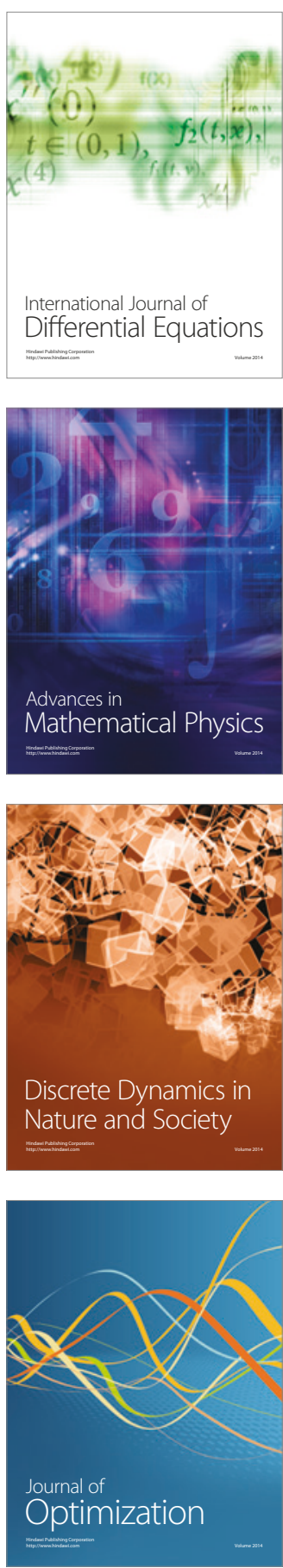